\title{
Semiclassical asymptotics for a class of singular Schrödinger operators
}

\author{
Rupert L. Frank \\ Simon Larson
}

To Ari Laptev on the occasion of his 70th birthday

\begin{abstract}
Let $\Omega \subset \mathbb{R}^{d}$ be bounded with $C^{1}$ boundary. In this paper we consider Schrödinger operators $-\Delta+W$ on $\Omega$ with $W(x) \approx \operatorname{dist}(x, \partial \Omega)^{-2}$ as $\operatorname{dist}(x, \partial \Omega) \rightarrow 0$. Under weak assumptions on $W$ we derive a two-term asymptotic formula for the sum of the eigenvalues of such operators.
\end{abstract}

Mathematics Subject Classification 2020. Primary 35P20

Keywords. Schrödinger operator, Semiclassical asymptotics.

\section{Introduction}

In this paper we consider semiclassical asymptotics for a class of Schrödinger operators on bounded sets $\Omega \subset \mathbb{R}^{d}$ with potentials which are singular at the boundary and subject to Dirichlet boundary conditions. Specifically, for a bounded open set $\Omega \subset \mathbb{R}^{d}$ with $C^{1}$ boundary we consider Schrödinger operators

$$
-\Delta+W(x) \quad \text { with } W(x) \approx \operatorname{dist}(x, \Omega)^{-2} \text { as } \operatorname{dist}(x, \partial \Omega) \rightarrow 0 .
$$

These operators have purely discrete spectrum and our main interest is towards the asymptotic behavior of their eigenvalues. Our main result is a two-term asymptotic formula for the sum of the eigenvalues.

Before we formulate our main result it is necessary to explain more precisely how (1.1) is to be interpreted. We shall assume that our potential decomposes as one part which is

R. L. Frank: Mathematisches Institut, Ludwig-Maximilans Universität München, Theresienstr. 39, 80333 München, Germany, and Department of Mathematics, California Institute of Technology, Pasadena, CA91125, USA; email: r.frank@lmu.de,rlfrank@caltech.edu

S. Larson: Department of Mathematics, California Institute of Technology, Pasadena, CA 91125, USA; email: larson@caltech.edu 
in $L_{\text {loc }}^{\infty}(\Omega)$ and has the prescribed singular behavior at the boundary and a part which, in comparison, is well-behaved. To simplify the exposition we write

$$
H_{\Omega, b, V}(h)=-h^{2} \Delta+h^{2}\left(b^{2}(x)-\frac{1}{4}\right) \frac{1}{\operatorname{dist}(x, \partial \Omega)^{2}}+h^{2} V(x)-1 \quad \text { for } h>0 .
$$

Technically, the operator $H_{\Omega, b, V}(h)$ is defined through the quadratic form

$$
u \mapsto \int_{\Omega}\left(h^{2}|\nabla u(x)|^{2}+h^{2}\left(b^{2}(x)-\frac{1}{4}\right) \frac{|u(x)|^{2}}{\operatorname{dist}(x, \partial \Omega)^{2}}+h^{2} V(x)|u(x)|^{2}-|u(x)|^{2}\right) d x
$$

with form domain $\left\{u \in H_{0}^{1}(\Omega): V_{+} u^{2} \in L^{1}(\Omega)\right\}$. Throughout we shall assume that $V \in L^{1}(\Omega), V_{-} \in L^{1+d / 2}(\Omega)$, and that $b \in L^{\infty}(\Omega)$ is positive and satisfies

$$
\lim _{r \rightarrow 0^{+}} \int_{\partial \Omega}\left[\sup _{y \in B_{r}(x) \cap \Omega} b(y)-\inf _{y \in B_{r}(x) \cap \Omega} b(y)\right] d \mathcal{H}^{d-1}(x)=0 .
$$

Here and in what follows we define $x_{ \pm}=\frac{|x| \pm x}{2}$ and note that with this convention both $x_{+}$ and $x_{-}$are non-negative. As a consequence of Hardy's inequality, the assumptions on $V$ and $b$ ensure that the quadratic form (1.2) is bounded from below and closed. Therefore it generates a selfadjoint, bounded from below operator $H_{\Omega, b, V}(h)$ in $L^{2}(\Omega)$.

We emphasize that by positivity of $b$, we mean $\inf _{\Omega} b>0$. This assumption can naturally be relaxed to require positivity only in a neighborhood of the boundary by adjusting $V$ correspondingly. The regularity assumption (1.3) implies that $\left.b\right|_{\partial \Omega}$ can be made sense of as an element of $L^{\infty}(\partial \Omega)$; indeed, by (1.3), $b$ has a well-defined limit $\mathcal{H}^{d-1}$-almost everywhere on $\partial \Omega$ which is finite since $b \in L^{\infty}(\Omega)$. Our main result can now be stated as follows:

Theorem 1.1. Let $\Omega \subset \mathbb{R}^{d}$ be open and bounded with $C^{1}$ boundary, $V \in L^{1}(\Omega)$ with $V_{-} \in L^{1+d / 2}(\Omega)$, and let $b \in L^{\infty}(\Omega)$ be positive and satisfy (1.3). Then, as $h \rightarrow 0^{+}$,

$$
\operatorname{Tr}\left(H_{\Omega, b, V}(h)\right)_{-}=L_{d} h^{-d}|\Omega|-\frac{L_{d-1}}{2} h^{-d+1} \int_{\partial \Omega} b(x) d \mathcal{H}^{d-1}(x)+o\left(h^{-d+1}\right),
$$

where $L_{d}=(4 \pi)^{-d / 2} \Gamma(2+d / 2)^{-1}$.

As a corollary of Theorem 1.1 we deduce:

Corollary 1.2. Let $\Omega \subset \mathbb{R}^{d}$ be open and bounded with $C^{1}$ boundary. Then, with $\Delta_{\Omega}$ denoting the Dirichlet Laplace operator in $\Omega$, as $h \rightarrow 0^{+}$and in the sense of measures

$$
\left.h^{d+1} \frac{\mathbb{1}\left(-h^{2} \Delta_{\Omega} \leq 1\right)(x, x)}{\operatorname{dist}(x, \partial \Omega)^{2}} d x \rightarrow \frac{L_{d-1}}{2} \mathcal{H}^{d-1}\right|_{\partial \Omega} .
$$

Proof. The corollary follows from a standard Feynman-Hellmann argument (cf. [10]) and Theorem 1.1 applied with the potential $W(x)=t f(x) / \operatorname{dist}(x, \partial \Omega)^{2}$ for $f \in C(\bar{\Omega})$ and sending first $h$ then $t$ to zero. 
Spectral asymptotics for differential operators that degenerate at the boundary of the domain are not new. However, the results in the literature mainly concern cases in which the operator degenerates at leading order and how this affects the first term in the asymptotics, see [1,2] and references therein. While the class of operators considered here is drastically less singular, our interest is towards the effect of the degeneracy on the second term in the asymptotics.

In the special case of the Dirichlet Laplacian, i.e. $V \equiv 0$ and $b \equiv 1 / 2$, Theorem 1.1 was proved in $[5,6]$. The strategy of our proof follows closely that developed there, but several new obstacles need to be circumvented in the presence of the potential, which is singular at the boundary. The idea is to localize the operator in balls whose size varies depending on the distance to the boundary and $h$. In a ball far from the boundary the influence of the boundary conditions and the potential both have a negligible effect and precise asymptotics can be obtained through standard methods. In a ball close to the boundary the regularity of the boundary allows to map the problem to a half-space where asymptotics are obtained by explicitly diagonalizing an effective operator. The main new ingredients needed here is to control how the straightening of the boundary affects the singular part of the potential and to understand how the potential enters in the half-space problem.

The works [5, 6] for domains with $C^{1}$ boundaries were extended to the case of Lipschitz boundaries in [7], see also [8]. Since the (weak) Hardy constant can be smaller than $1 / 4$ for Lipschitz domains, it is not clear how to generalize the results of the present paper to this setting.

The plan for the paper is as follows. In Section 2 we recall a number of results concerning changes of variables mapping $\partial \Omega$ locally to a hyperplane. In particular, Lemma 2.2 describes how such a mapping affects the singular part of our potential. We also prove a local Hardy-Lieb-Thirring inequality which will be crucial in controlling error terms appearing in our analysis, and which replaces the Lieb-Thirring inequality in [5] in the absence of a singular potential. In Section 3 we provide local asymptotics, both in the bulk of our domain and close to the boundary. Finally, in Section 4 we adapt the localization procedure developed in $[5,6,7]$ to our current setting and use it to piece together the local asymptotics of Section 3, thus proving Theorem 1.1.

The letter $C$ will denote a constant whose value can change at each occurence.

We are deeply grateful to Ari Laptev for sharing his fascination for spectral estimates and Hardy's inequality with us and we would like to dedicate this paper to him on the occasion of his 70th birthday.

\section{Preliminaries}

\subsection{Straightening the boundary}

Let $\mathbb{R}_{+}^{d}=\left\{y \in \mathbb{R}^{d}: y_{d}>0\right\}$. Let $B \subset \mathbb{R}^{d}$ be an open ball of radius $\ell$ centred at a point $x_{0} \in \partial \Omega$. By rotating and translating we may assume that $x_{0}=0$ and $v_{0}=(0, \ldots, 0,1)$ 
is the inward pointing unit normal to $\partial \Omega$ at $x_{0}$. Since $\Omega$ is bounded with $C^{1}$ boundary, there exists a non-decreasing modulus of continuity $\omega: \mathbb{R}_{+} \rightarrow[0,1]$ such that, if $\ell$ is small enough, there exists a function $f: \mathbb{R}^{d-1} \rightarrow \mathbb{R}$ satisfying $\left|\nabla f\left(x^{\prime}\right)\right| \leq \omega\left(\left|x^{\prime}\right|\right)$ such that

$$
\partial \Omega \cap B_{2 \ell}(0)=\left\{\left(x^{\prime}, x_{d}\right) \in \mathbb{R}^{d-1} \times \mathbb{R}: x_{d}=f\left(x^{\prime}\right)\right\} \cap B_{2 \ell}(0) .
$$

Note that, by the choice of coordinates, $f(0)=0$ and $\nabla f(0)=0$.

Set $\mathcal{X}=\left\{\left(x^{\prime}, x_{d}\right) \in \mathbb{R}^{d-1} \times \mathbb{R}:\left|x^{\prime}\right|<2 \ell\right\}$. We define a diffeomorphism $\Phi: \mathcal{X} \rightarrow \mathbb{R}^{d}$ by $\Phi_{j}(x)=x_{j}$ for $j=1, \ldots, d-1$ and $\Phi_{d}(x)=x_{d}-f\left(x^{\prime}\right)$. Note that the Jacobian determinant of $\Phi$ equals 1 and that the inverse of $\Phi$ is well-defined on $\Phi(\mathcal{X})=\mathcal{X}$. The inverse is given by $\Phi_{j}^{-1}(y)=y_{j}$ for $j=1, \ldots, d-1$ and $\Phi_{d}^{-1}(y)=y_{d}+f\left(y^{\prime}\right)$.

In the following lemma we gather some results whose proofs are standard and can be found, for instance, in [6, Section 4].

Lemma 2.1 (Straightening of the boundary). Let $B, \Phi$ be as above and for $u: B \rightarrow \mathbb{R}$ set $\tilde{u}=u \circ \Phi^{-1}$. For $0<\ell \leq c(\omega)$ and with $C$ depending only on $d$, we have:

1. if $u \in L^{1}(B)$ then

$$
\int_{B} u(x) d x=\int_{\Phi(B)} \tilde{u}(y) d y .
$$

2. if $u \in L^{\infty}(\partial \Omega \cap B)$ then

$$
\left|\int_{\partial \Omega \cap B} u(x) d \mathcal{H}^{d-1}(x)-\int_{\partial \mathbb{R}_{+}^{d} \cap \Phi(B)} \tilde{u}(y) d \mathcal{H}^{d-1}(y)\right| \leq C \ell^{d-1} \omega(\ell)^{2}\|u\|_{L^{\infty}} .
$$

3. if $u \in H_{0}^{1}(\Omega \cap B)$ then $\tilde{u} \in H_{0}^{1}\left(\mathbb{R}_{+}^{d} \cap \Phi(B)\right)$ and

$$
\left.\left|\int_{\Omega \cap B}\right| \nabla u(x)\right|^{2} d x-\left.\int_{\mathbb{R}_{+}^{d} \cap \Phi(B)}|\nabla \tilde{u}(y)|^{2} d y\left|\leq C \omega(\ell) \int_{\mathbb{R}_{+}^{d} \cap \Phi(B)}\right| \nabla \tilde{u}(y)\right|^{2} d y .
$$

4. if $u \in C_{0}^{1}\left(\mathbb{R}^{d}\right)$ is supported in $\bar{B}$ then, after extension by zero, $\tilde{u} \in C_{0}^{1}\left(\mathbb{R}^{d}\right)$ with $\operatorname{supp} \tilde{u} \subseteq \overline{B_{2 \ell}(0)}$ and $\|\nabla \tilde{u}\|_{L^{\infty}} \leq C\|\nabla u\|_{L^{\infty}}$.

In addition to the properties in Lemma 2.1 we will need the following result which enables us to control the change of the singular part of our potentials:

Lemma 2.2. Let $B, \Phi$ be as above. There is a constant $C$ depending only on $d$ such that for any $x \in B \cap \Omega$,

$$
0 \leq \frac{1}{\operatorname{dist}(x, \partial \Omega)^{2}}-\frac{1}{\operatorname{dist}\left(\Phi(x), \partial \mathbb{R}_{+}^{d}\right)^{2}} \leq C \frac{\omega(2 \ell)^{2}}{\operatorname{dist}\left(\Phi(x), \partial \mathbb{R}_{+}^{d}\right)^{2}} .
$$

Proof. By definition of $f,\left(x^{\prime}, f\left(x^{\prime}\right)\right) \in \partial \Omega$, thus $\operatorname{dist}(x, \partial \Omega) \leq\left|x-\left(x^{\prime}, f\left(x^{\prime}\right)\right)\right|=$ $\left|x_{d}-f\left(x^{\prime}\right)\right|=\operatorname{dist}\left(\Phi(x), \partial \mathbb{R}_{+}^{d}\right)$ which implies the lower bound in (2.1). 
To prove the upper bound, let $z=\left(z^{\prime}, f\left(z^{\prime}\right)\right) \in \partial \Omega$ be such that $\operatorname{dist}(x, \partial \Omega)=|x-z|$. Since $\partial \Omega$ is parametrized by $f$ in the larger ball $B_{2 \ell}\left(x_{0}\right)$ it is clear that such a point exists and that $z \in B_{2 \ell}\left(x_{0}\right)$. The point $z$ might not be uniquely determined but that will not play any role in what follows.

We begin by rewriting the expression we want to bound in terms of $z$ :

$$
\begin{aligned}
\frac{1}{\operatorname{dist}(x, \partial \Omega)^{2}}-\frac{1}{\operatorname{dist}\left(\Phi(x), \partial \mathbb{R}_{+}^{d}\right)^{2}} & =\frac{1}{|x-z|^{2}}-\frac{1}{\left|x_{d}-f\left(x^{\prime}\right)\right|^{2}} \\
& =\frac{\left(f\left(x^{\prime}\right)-f\left(z^{\prime}\right)\right)\left(f\left(x^{\prime}\right)+f\left(z^{\prime}\right)-2 x_{d}\right)-\left|x^{\prime}-z^{\prime}\right|^{2}}{|x-z|^{2}\left|x_{d}-f\left(x^{\prime}\right)\right|^{2}} .
\end{aligned}
$$

Since $f$ is $C^{1}$ and by the definition of $z$ it holds that

$$
x=z+|x-z| \frac{\left(-\nabla f\left(z^{\prime}\right), 1\right)}{\sqrt{1+\left|\nabla f\left(z^{\prime}\right)\right|^{2}}} .
$$

Consequently,

$$
\left|x^{\prime}-z^{\prime}\right|^{2}=|x-z|^{2} \frac{\left|\nabla f\left(z^{\prime}\right)\right|^{2}}{1+\left|\nabla f\left(z^{\prime}\right)\right|^{2}} \quad \text { and } \quad\left|x_{d}-f\left(z^{\prime}\right)\right|^{2}=\frac{|x-z|^{2}}{1+\left|\nabla f\left(z^{\prime}\right)\right|^{2}} .
$$

Note also that $f\left(x^{\prime}\right) \leq f\left(z^{\prime}\right) \leq x_{d}$. From the above identities one finds

$$
\begin{aligned}
\frac{1}{\operatorname{dist}(x, \partial \Omega)^{2}}-\frac{1}{\operatorname{dist}\left(\Phi(x), \partial \mathbb{R}_{+}^{d}\right)^{2}}= & \frac{1}{\left|x_{d}-f\left(x^{\prime}\right)\right|^{2}}\left[\frac{\left|f\left(x^{\prime}\right)-f\left(z^{\prime}\right)\right|^{2}}{|x-z|^{2}}\right. \\
& \left.+2 \frac{\left|f\left(x^{\prime}\right)-f\left(z^{\prime}\right)\right|}{|x-z| \sqrt{1+\left|\nabla f\left(z^{\prime}\right)\right|^{2}}}-\frac{\left|\nabla f\left(z^{\prime}\right)\right|^{2}}{1+\left|\nabla f\left(z^{\prime}\right)\right|^{2}}\right] .
\end{aligned}
$$

By the fundamental theorem of calculus and (2.2)

$$
\left|f\left(x^{\prime}\right)-f\left(z^{\prime}\right)\right|=\left|\left(x^{\prime}-z^{\prime}\right) \int_{0}^{1} \nabla f\left(t x^{\prime}+(1-t) z^{\prime}\right) d t\right| \leq \omega(2 \ell)^{2}|x-z| .
$$

Therefore

$$
\frac{\left|f\left(x^{\prime}\right)-f\left(z^{\prime}\right)\right|^{2}}{|x-z|^{2}}+2 \frac{\left|f\left(x^{\prime}\right)-f\left(z^{\prime}\right)\right|}{|x-z| \sqrt{1+\left|\nabla f\left(z^{\prime}\right)\right|^{2}}}-\frac{\left|\nabla f\left(z^{\prime}\right)\right|^{2}}{1+\left|\nabla f\left(z^{\prime}\right)\right|^{2}} \leq C \omega(2 \ell)^{2} .
$$

Combined with (2.3) this completes the proof of Lemma 2.2.

\subsection{A local Hardy-Lieb-Thirring inequality}

The aim of this subsection is to prove a bound for localized traces of our operator. Before stating the result we recall the following Hardy inequality due to Davies [3] (combine his Theorems 2.3 and 2.4). 
Lemma 2.3. Let $\Omega \subset \mathbb{R}^{d}$ be open and bounded with $C^{1}$-boundary. Then for any $\varepsilon>0$ there is a $c_{H}(\varepsilon, \Omega) \geq 0$ such that for all $u \in H_{0}^{1}(\Omega)$,

$$
\int_{\Omega}|\nabla u(x)|^{2} d x+\left(\varepsilon-\frac{1}{4}\right) \int_{\Omega} \frac{|u(x)|^{2}}{\operatorname{dist}(x, \partial \Omega)^{2}} d x \geq-c_{H}(\varepsilon, \Omega) \int_{\Omega}|u(x)|^{2} d x .
$$

Remark. Lemma 2.3 can be proved in a direct manner by using a partition of unity and appealing to Lemmas 2.1 and 2.2. In particular, this allows one to quantify the best constant $c_{H}$ in terms of the $C^{1}$-regularity of $\partial \Omega$. Indeed, such a proof yields the bound $c_{H}(\varepsilon, \Omega) \leq \frac{C}{\omega^{-1}(\varepsilon)^{2}}$ for a constant $C$ depending only on the dimension and $\omega^{-1}$ is the inverse of the $C^{1}$-modulus of continuity of $\partial \Omega$.

With Lemma 2.3 in hand we move on to the main result of this subsection. Specifically, the following local Hardy-Lieb-Thirring type inequality for $H_{\Omega, b, V}$ (cf. [9]):

Lemma 2.4. Let $\Omega, b, V$ be as in Theorem 1.1. Let $\phi \in C_{0}^{1}\left(\mathbb{R}^{d}\right)$ be supported in a ball $\bar{B}$ of radius $\ell$ and set $\underline{b}=\inf _{\Omega \cap B} b$. If $0<h \leq K \min \left\{\ell, c_{H}\left(\underline{b}^{2} / 2, \Omega\right)^{-1 / 2}\right\}$, then

$$
\operatorname{Tr}\left(\phi H_{\Omega, b, V}(h) \phi\right)_{-} \leq C \min \{\underline{b}, 1\}^{-d} \ell^{d} h^{-d}\left(1+h^{2}\left\|V_{-}\right\|_{L^{1+d / 2}(\Omega \cap B)}^{1+d / 2}\right),
$$

where the constant $C$ depends only on $d, K$, and $\|\phi\|_{L^{\infty}}$.

Proof of Lemma 2.4. By assumption, $\underline{b}>0$. By the variational principle and for any $\delta \in(0,1 / 2]$, we find

$$
\begin{aligned}
\phi H_{\Omega, b, V}(h) \phi \geq & \phi\left(-h^{2} \delta \Delta-h^{2} V_{-}(x)-1\right. \\
& \left.-h^{2}(1-\delta)\left(-\Delta+(1-\delta)^{-1}\left(\underline{b}^{2}-\frac{1}{4}\right) \frac{1}{\operatorname{dist}(x, \partial \Omega)^{2}}\right)\right) \phi .
\end{aligned}
$$

Since $\delta \in(0,1 / 2]$ we have

$$
(1-\delta)^{-1}\left(\underline{b}^{2}-\frac{1}{4}\right) \geq(1+2 \delta)\left(\underline{b}^{2}-\frac{1}{4}\right)>\underline{b}^{2}-\frac{\delta}{2}-\frac{1}{4} .
$$

Thus, setting $\delta=\min \left\{\underline{b}^{2}, 1 / 2\right\} \leq 1 / 2$, Lemma 2.3 implies with $c_{0}=c_{H}\left(\underline{b}^{2} / 2, \Omega\right)$ that

$$
\phi H_{\Omega, b, V}(h) \phi \geq \phi\left(-h^{2} \delta \Delta-c_{0} h^{2}-h^{2} V_{-}(x)-1\right) \phi \text {. }
$$

Consequently, for any $0<\rho<1$, the variational principle and (2.4) yields

$$
\begin{aligned}
\operatorname{Tr}\left(\phi H_{\Omega, b, V}(h) \phi\right)_{-} \leq & \operatorname{Tr}\left(\phi\left(-h^{2} \delta(1-\rho) \Delta-c_{0} h^{2}-1\right) \phi\right)_{-} \\
& +\operatorname{Tr}\left(\phi\left(-h^{2} \delta \rho \Delta-h^{2} V_{-}\right) \phi\right)_{-} .
\end{aligned}
$$

Using the Berezin-Li-Yau inequality

$$
\begin{aligned}
\operatorname{Tr}\left(\phi \left(-h^{2} \delta(1-\rho) \Delta\right.\right. & \left.\left.-c_{0} h^{2}-1\right) \phi\right)_{-} \\
& \leq C\left(1+c_{0} h^{2}\right)^{1+d / 2}(1-\rho)^{-d / 2} \delta^{-d / 2} h^{-d} \ell^{d},
\end{aligned}
$$


with $C>0$ depending on $d$ and $\|\phi\|_{L^{\infty}}$. For the remaining term the Lieb-Thirring inequality implies

$$
\operatorname{Tr}\left(\phi\left(-h^{2} \delta \rho \Delta-h^{2} V_{-}\right) \phi\right)_{-} \leq C h^{2} \delta^{-d / 2} \rho^{-d / 2}\left\|V_{-}\right\|_{L^{1+d / 2}\left(\Omega \cap B_{\ell}\right)}^{1+d / 2},
$$

for some $C>0$ depending only on $d$. Gathering the estimates and setting $\rho=$ $h^{2} /\left(2 K^{2} \ell^{2}\right)<1$ completes the proof.

\section{Local asymptotics}

\subsection{Local asymptotics in the bulk}

Lemma 3.1. Let $\phi \in C_{0}^{1}\left(\mathbb{R}^{d}\right)$ be supported in a ball $\bar{B}$ of radius $\ell>0$ and satisfy

$$
\|\nabla \phi\|_{L^{\infty}\left(\mathbb{R}^{d}\right)} \leq M \ell^{-1}
$$

If $V \in L^{1}(B)$ is such that $V_{-}=V_{0}+V_{1}$ with $0 \leq V_{0} \in L^{\infty}(B)$ and $V_{1} \in L^{1+d / 2}(B)$ then, for $0<h \leq K \min \left\{\ell,\left\|V_{0}\right\|_{\infty}^{-1 / 2}\right\}$,

$$
\begin{aligned}
& \left|\operatorname{Tr}\left(\phi\left(-h^{2} \Delta+h^{2} V-1\right) \phi\right)_{-}-L_{d} h^{-d} \int_{B} \phi^{2}(x) d x\right| \\
& \leq C h^{-d+2}\left[\ell^{d-2}+\ell^{d}\left\|V_{0}\right\|_{L^{\infty}(B)}+\ell^{d}\left\|V_{1}\right\|_{L^{1+d / 2}(B)}^{1+d / 2}+\left\|V_{+}\right\|_{L^{1}(B)}\right],
\end{aligned}
$$

where the constant $C$ depends only on $d, M, K$.

Proof. Throughout the proof we set $H_{V}=H_{\mathbb{R}^{d}, 0, V}=-h^{2} \Delta+h^{2} V-1$ in $L^{2}\left(\mathbb{R}^{d}\right)$.

To prove the lower bound, consider the operator $\gamma$ with integral kernel

$$
\gamma(x, y)=\frac{1}{(2 \pi)^{d}} \chi(x) \int_{|\xi|<h^{-1}} e^{i \xi(x-y)} d \xi \chi(y),
$$

where $\chi \in C_{0}^{\infty}\left(\mathbb{R}^{d}\right)$ with $0 \leq \chi \leq 1$ and $\chi \equiv 1$ on $B$. The operator $\gamma$ is trace class and satisfies $0 \leq \gamma \leq \mathbf{1}$. Therefore, the variational principle implies that

$$
\begin{aligned}
\operatorname{Tr}\left(\phi H_{V} \phi\right)_{-} & \geq \operatorname{Tr}\left(\phi H_{V_{+}} \phi\right)_{-} \\
& \geq-\operatorname{Tr}\left(\gamma \phi H_{V_{+}} \phi\right) \\
& =-\frac{1}{(2 \pi)^{d}} \int_{|\xi|<h^{-1}}\left(h^{2} \| \nabla e^{\left.i \xi \cdot \phi\left\|_{L^{2}\left(\mathbb{R}^{d}\right)}^{2}+h^{2}\right\| V_{+} \phi^{2}\left\|_{L^{1}\left(\mathbb{R}^{d}\right)}-\right\| \phi \|_{L^{2}\left(\mathbb{R}^{d}\right)}^{2}\right) d \xi}\right. \\
& =L_{d} h^{-d} \int_{B} \phi^{2}(x) d x-C h^{-d+2}\left(\|\nabla \phi\|_{L^{2}\left(\mathbb{R}^{d}\right)}^{2}+\left\|V_{+} \phi^{2}\right\|_{L^{1}\left(\mathbb{R}^{d}\right)}\right) .
\end{aligned}
$$

Since, by (3.1), $\|\phi\|_{L^{\infty}} \leq M$ and $\|\nabla \phi\|_{L^{2}\left(\mathbb{R}^{d}\right)}^{2} \leq C \ell^{d-2}$ this proves the lower bound. 
It remains to prove the upper bound. For any $0<\rho \leq 1 / 2$

$$
\begin{aligned}
\operatorname{Tr}\left(\phi H_{V} \phi\right)_{-} & \leq \operatorname{Tr}\left(\phi H_{V_{-}} \phi\right)_{-} \\
& \leq \operatorname{Tr}\left(\phi\left(-h^{2}(1-\rho) \Delta-h^{2} V_{0}-1\right) \phi\right)_{-}+h^{2} \operatorname{Tr}\left(\phi\left(-\rho \Delta-V_{1}\right) \phi\right)_{-} .
\end{aligned}
$$

To bound the second term we apply the Lieb-Thirring inequality to conclude that

$$
h^{2} \operatorname{Tr}\left(\phi\left(-\rho \Delta-V_{1}\right) \phi\right)_{-} \leq h^{2} \operatorname{Tr}\left(\phi\left(-\rho \Delta-V_{1} 1_{B}\right)_{-} \phi\right) \leq C h^{2} \rho^{-d / 2} \int_{B}\left|V_{1}(x)\right|^{1+d / 2} d x,
$$

where we again used $\|\phi\|_{L^{\infty}} \leq M$. Since $V_{0} \in L^{\infty}(B)$, we can bound

$$
\begin{aligned}
\operatorname{Tr}\left(\phi\left(-h^{2}(1-\rho) \Delta-h^{2} V_{0}-1\right) \phi\right)_{-} & \leq \operatorname{Tr}\left(\phi\left(-h^{2}(1-\rho) \Delta-h^{2} \sup _{B} V_{0}-1\right) \phi\right)_{-} \\
& =\left(1+h^{2} \sup _{B} V_{0}\right) \operatorname{Tr}\left(\phi\left(-\tilde{h}^{2} \Delta-1\right) \phi\right)_{-}
\end{aligned}
$$

with $\tilde{h}=h(1-\rho)^{1 / 2}\left(1+h^{2} \sup _{B} V_{0}\right)^{-1 / 2}$. By the Berezin-Li-Yau inequality,

$$
\operatorname{Tr}\left(\phi\left(-\tilde{h}^{2} \Delta-1\right) \phi\right)_{-} \leq L_{d} \tilde{h}^{-d} \int_{B} \phi^{2}(x) d x .
$$

Combining the above we have arrived at

$$
\begin{aligned}
\operatorname{Tr}\left(\phi H_{V} \phi\right)_{-} \leq & L_{d} h^{-d} \int_{B} \phi^{2}(x) d x+C h^{2} \rho^{-d / 2} \int_{B}\left|V_{1}(x)\right|^{1+d / 2} d x \\
& +L_{d} h^{-d}\left[(1-\rho)^{-d / 2}\left(1+h^{2} \sup _{B} V_{0}\right)^{1+d / 2}-1\right] \int_{B} \phi^{2}(x) d x \\
\leq & L_{d} h^{-d} \int_{B} \phi^{2}(x) d x+C h^{2} \rho^{-d / 2} \int_{B}\left|V_{1}(x)\right|^{1+d / 2} d x \\
& +C h^{-d}\left[\rho+h^{2} \sup _{B} V_{0}\right] \int_{B} \phi^{2}(x) d x,
\end{aligned}
$$

where $C$ depend only on $d, K, M$. Setting $\rho=h^{2} /\left(2 K^{2} \ell^{2}\right) \leq 1 / 2$ and using $\int \phi^{2} \leq C \ell^{d}$ completes the proof.

\subsection{Local asymptotics near the boundary}

In this section we prove the following local asymptotic expansion close to the boundary:

Theorem 3.2. Let $\Omega, b, V$ be as in Theorem 1.1. Let $\phi \in C_{0}^{1}\left(\mathbb{R}^{d}\right)$ be supported in a ball $\bar{B}$ of radius $\ell$ and satisfy

$$
\|\nabla \phi\|_{L^{\infty}\left(\mathbb{R}^{d}\right)} \leq M \ell^{-1}
$$


Assume that $\operatorname{dist}(B, \partial \Omega) \leq 2 \ell$, and set $\underline{b}=\inf _{B \cap \Omega} b$. For $0<\ell \leq c(\Omega, \underline{b})$ and $0<h \leq$ $K \ell$,

$$
\begin{aligned}
& \left|\operatorname{Tr}\left(\phi H_{\Omega, b, V}(h) \phi\right)_{-}-L_{d} h^{-d} \int_{\Omega} \phi^{2}(x) d x+\frac{L_{d-1}}{2} h^{-d+1} \int_{\partial \Omega} \phi^{2}(x) b(x) d \mathcal{H}^{d-1}(x)\right| \\
& \leq \ell^{d} h^{-d}{ } \ell \rightarrow 0^{+}(1)+O\left(h^{-d+1}\right) \int_{\partial \Omega} \phi^{2}(x)\left[\sup _{y \in B_{2 \ell}(x)} b(y)-\inf _{y \in B_{2 \ell}(x)} b(y)\right] d \mathcal{H}^{d-1}(x) \\
& \quad+O\left(h^{-d+2}\right)\left(\ell^{d-2} \log (\ell / h)+\left\|V_{+}\right\|_{L^{1}(\Omega \cap B)}+\ell^{d}\left\|V_{-}\right\|_{L^{1+d / 2}(\Omega \cap B)}^{1+d / 2}\right)
\end{aligned}
$$

Moreover, the error terms and the implicit constants can be quantified in terms of the $C^{1}$-regularity of $\partial \Omega$ and $M, K,\|b\|_{L^{\infty}(\Omega \cap B)}, \underline{b}$.

The proof of Theorem 3.2 will be split into several lemmas. The first of which reduces our problem to the corresponding in a half-space:

Lemma 3.3. Let $\Omega, b, V$ be as in Theorem 1.1. Let $\phi \in C_{0}^{1}\left(\mathbb{R}^{d}\right)$ be supported in a ball $\bar{B}$ of radius $\ell$ such that $\operatorname{dist}(B, \partial \Omega) \leq 2 \ell$, and $\inf _{B \cap \Omega} b=\underline{b}>0$. For $0<\ell \leq c(\Omega, \underline{b})$ and $0<h \leq K \ell$ with $\tilde{\phi}=\phi \circ \Phi^{-1}, \tilde{V}=V \circ \Phi^{-1}$,

$$
\begin{aligned}
\operatorname{Tr}\left(\tilde{\phi} H_{\mathbb{R}_{+}^{d}, \bar{b}, \tilde{V}}(h) \tilde{\phi}\right)_{-}-\ell^{d} h^{-d} o_{\ell \rightarrow 0^{+}}(1)\left(1+h^{2}\left\|V_{-}\right\|_{L^{1+d / 2}(\Omega \cap B)}^{1+d / 2}\right) \\
\leq \operatorname{Tr}\left(\phi H_{\Omega, b, V}(h) \phi\right)_{-} \\
\leq \operatorname{Tr}\left(\tilde{\phi} H_{\mathbb{R}_{+}^{d}, \underline{b}, \tilde{V}}(h) \tilde{\phi}\right)_{-}+\ell^{d} h^{-d} o_{\ell \rightarrow 0^{+}}(1)\left(1+h^{2}\left\|V_{-}\right\|_{L^{1+d / 2}(\Omega \cap B)}^{1+d / 2}\right)
\end{aligned}
$$

where $\bar{b}=\sup _{x \in B \cap \Omega} b(x)$. Moreover, the error terms and the implicit constants can be quantified in terms of the $C^{1}$-regularity of $\partial \Omega$ and $K, \underline{b}, \bar{b},\|\phi\|_{L^{\infty}}$.

Proof. Provided $\ell$ is small enough there exists a ball $B^{\prime} \supset B$ with centre on $\partial \Omega$ and radius $4 \ell$ which satisfies the assumptions in Section 2.1. Let $\Phi$ be the associated diffeomorphism.

We split the proof into two parts, in the first part we prove the upper bound and in the second we prove the lower bound.

Part 1: (Proof of the upper bound) By the variational principle

$$
\operatorname{Tr}\left(\phi H_{\Omega, b, V}(h) \phi\right)_{-} \leq \operatorname{Tr}\left(\phi H_{\Omega, \underline{b}, V}(h) \phi\right)_{-} .
$$

Moreover, by Lemma 2.1 there exists $C_{0}>0$ depending only on $d$ such that

$$
\operatorname{Tr}\left(\phi H_{\Omega, \underline{b}, V}(h) \phi\right)_{-} \leq \operatorname{Tr}\left(\tilde{\phi}\left(-h^{2}\left(1-C_{0} \omega(4 \ell)\right) \Delta_{\mathbb{R}_{+}^{d}}+h^{2} \frac{\underline{b}^{2}-1 / 4}{\operatorname{dist}\left(\Phi^{-1}(\cdot), \partial \Omega\right)^{2}}+h^{2} \tilde{V}-1\right) \tilde{\phi}\right)_{-} .
$$

We claim that

$$
\begin{aligned}
& \operatorname{Tr}\left(\tilde{\phi}\left(-h^{2}\left(1-C_{0} \omega(4 \ell)\right) \Delta_{\mathbb{R}_{+}^{d}}+h^{2} \frac{\underline{b}^{2}-1 / 4}{\operatorname{dist}\left(\Phi^{-1}(\cdot), \partial \Omega\right)^{2}}+h^{2} \tilde{V}-1\right) \tilde{\phi}\right)_{-} \\
& \quad \leq \operatorname{Tr}\left(\tilde{\phi}\left(-h^{2}\left(1-C_{0} \omega(4 \ell)\right) \Delta_{\mathbb{R}_{+}^{d}}+h^{2} \frac{\underline{b}^{2}-1 / 4-C \omega(8 \ell)^{2}}{\operatorname{dist}\left(\cdot, \partial \mathbb{R}_{+}^{d}\right)^{2}}+h^{2} \tilde{V}-1\right) \tilde{\phi}\right)_{-}
\end{aligned}
$$


for a constant $C$ depending only on $d$. Indeed, if $\underline{b} \geq 1 / 2$ Lemma 2.2 and the variational principle implies

$$
\begin{aligned}
& \operatorname{Tr}\left(\tilde{\phi}\left(-h^{2}\left(1-C_{0} \omega(4 \ell)\right) \Delta_{\mathbb{R}_{+}^{d}}+h^{2} \frac{\underline{b}^{2}-1 / 4}{\operatorname{dist}\left(\Phi^{-1}(\cdot), \partial \Omega\right)^{2}}+h^{2} \tilde{V}-1\right) \tilde{\phi}\right)_{-} \\
& \quad \leq \operatorname{Tr}\left(\tilde{\phi}\left(-h^{2}\left(1-C_{0} \omega(4 \ell)\right) \Delta_{\mathbb{R}_{+}^{d}}+h^{2} \frac{\underline{b}^{2}-1 / 4}{\operatorname{dist}\left(\cdot, \partial \mathbb{R}_{+}^{d}\right)^{2}}+h^{2} \tilde{V}-1\right) \tilde{\phi}\right)_{-} \\
& \quad \leq \operatorname{Tr}\left(\tilde{\phi}\left(-h^{2}\left(1-C_{0} \omega(4 \ell)\right) \Delta_{\mathbb{R}_{+}^{d}}+h^{2} \frac{\underline{b}^{2}-1 / 4-C \omega(8 \ell)^{2}}{\operatorname{dist}\left(\cdot, \partial \mathbb{R}_{+}^{d}\right)^{2}}+h^{2} \tilde{V}-1\right) \tilde{\phi}\right)_{-}
\end{aligned}
$$

Similarly, if $0<\underline{b}<1 / 2$ Lemma 2.2 and the variational principle implies

$$
\begin{aligned}
& \operatorname{Tr}\left(\tilde{\phi}\left(-h^{2}\left(1-C_{0} \omega(4 \ell)\right) \Delta_{\mathbb{R}_{+}^{d}}+h^{2} \frac{\underline{b}^{2}-1 / 4}{\operatorname{dist}\left(\Phi^{-1}(\cdot), \partial \Omega\right)^{2}}+h^{2} \tilde{V}-1\right) \tilde{\phi}\right)_{-} \\
& \quad \leq \operatorname{Tr}\left(\tilde{\phi}\left(-h^{2}\left(1-C_{0} \omega(4 \ell)\right) \Delta_{\mathbb{R}_{+}^{d}}+h^{2} \frac{\left(\underline{b}^{2}-1 / 4\right)\left(1+C \omega(8 \ell)^{2}\right)}{\operatorname{dist}\left(\cdot, \partial \mathbb{R}_{+}^{d}\right)^{2}}+h^{2} \tilde{V}-1\right) \tilde{\phi}\right)_{-} \\
& \quad \leq \operatorname{Tr}\left(\tilde{\phi}\left(-h^{2}\left(1-C_{0} \omega(4 \ell)\right) \Delta_{\mathbb{R}_{+}^{d}}+h^{2} \frac{\underline{b}^{2}-1 / 4-C \omega(8 \ell)^{2}}{\operatorname{dist}\left(\cdot, \partial \mathbb{R}_{+}^{d}\right)^{2}}+h^{2} \tilde{V}-1\right) \tilde{\phi}\right)_{-} .
\end{aligned}
$$

For any $2 C_{0} \omega(4 \ell)<\rho \leq 1 / 2$ we estimate

$$
\begin{aligned}
& \operatorname{Tr}\left(\tilde{\phi}\left(-h^{2}\left(1-C_{0} \omega(4 \ell)\right) \Delta_{\mathbb{R}_{+}^{d}}+h^{2} \frac{\underline{b}^{2}-1 / 4-C \omega(8 \ell)^{2}}{\operatorname{dist}\left(\cdot, \partial \mathbb{R}_{+}^{d}\right)^{2}}+h^{2} \tilde{V}-1\right) \tilde{\phi}\right)_{-} \\
& \leq \operatorname{Tr}\left(\tilde{\phi} H_{\mathbb{R}_{+}^{d}, \underline{b}, \tilde{V}}(h) \tilde{\phi}\right)_{-} \\
& \quad+\operatorname{Tr}\left(\tilde{\phi}\left(-h^{2}\left(\rho-C_{0} \omega(4 \ell)\right) \Delta_{\mathbb{R}_{+}^{d}}+h^{2} \frac{\rho\left(\underline{b}^{2}-1 / 4\right)-C \omega(8 \ell)^{2}}{\operatorname{dist}\left(\cdot, \partial \mathbb{R}_{+}^{d}\right)^{2}}+h^{2} \rho \tilde{V}-\rho\right) \tilde{\phi}\right)_{-} .
\end{aligned}
$$

Provided

$$
\frac{\rho\left(\underline{b}^{2}-1 / 4\right)-C \omega(8 \ell)^{2}}{\rho-C_{0} \omega(4 \ell)}=\left(\underline{b}^{2}-1 / 4\right) \frac{1}{1-C_{0} \omega(4 \ell) \rho^{-1}}-C \frac{\omega(8 \ell)^{2}}{\rho-C_{0} \omega(4 \ell)}>-\frac{1}{4},
$$

we can apply the local Hardy-Lieb-Thirring inequality of Lemma 2.4 in $\mathbb{R}_{+}^{d}$ to bound

$$
\begin{aligned}
& \operatorname{Tr}\left(\tilde{\phi}\left(-h^{2}\left(\rho-C_{0} \omega(4 \ell)\right) \Delta_{\mathbb{R}_{+}^{d}}+h^{2} \frac{\rho\left(\underline{b}^{2}-1 / 4\right)-C \omega(8 \ell)^{2}}{\operatorname{dist}\left(\cdot, \partial \mathbb{R}_{+}^{d}\right)^{2}}+h^{2} \rho \tilde{V}-\rho\right) \tilde{\phi}\right)_{-} \\
& \quad \leq C \rho^{1+d / 2} \ell^{d} h^{-d}\left(\rho-C_{0} \omega(4 \ell)\right)^{-d / 2}\left(1+h^{2} \rho^{d / 2}\left(\rho-C_{0} \omega(4 \ell)\right)^{-d / 2}\left\|V_{-}\right\|_{L^{1+d / 2}(\Omega \cap B)}^{1+d / 2}\right) \\
& \quad \leq C \rho \ell^{d} h^{-d}\left(1+h^{2}\left\|V_{-}\right\|_{L^{1+d / 2}(\Omega \cap B)}^{1+d / 2}\right) .
\end{aligned}
$$


Set $\rho=\sqrt{\omega(4 \ell)}+\omega(8 \ell)$. Then $\rho>2 C_{0} \omega(4 \ell)$ and (3.2) are valid provided $\ell$ is small enough. Therefore, upon collecting the estimates above we arrive at the bound

$$
\begin{aligned}
\operatorname{Tr}\left(\phi H_{\Omega, b, V}(h) \phi\right)_{-} \leq & \operatorname{Tr}\left(\tilde{\phi} H_{\mathbb{R}_{+}^{d}, \underline{b}, \tilde{V}}(h) \tilde{\phi}\right)_{-} \\
& +C \ell^{d} h^{-d}(\sqrt{\omega(4 \ell)}+\omega(8 \ell))\left(1+h^{2}\left\|V_{-}\right\|_{L^{1+d / 2}(\Omega \cap B)}^{1+d / 2}\right),
\end{aligned}
$$

thus completing the proof of the upper bound.

Part 2: (Proof of the lower bound) The proof of the lower bound proceeds as the upper bound but with the roles of $\Omega$ and $\mathbb{R}_{+}^{d}$ exchanged.

By Lemma 2.1,

$\operatorname{Tr}\left(\tilde{\phi} H_{\mathbb{R}_{+}^{d}, \bar{b}, \tilde{V}}(h) \tilde{\phi}\right)_{-} \leq \operatorname{Tr}\left(\phi\left(-h^{2}\left(1+C_{0} \omega(4 \ell)\right)^{-1} \Delta_{\Omega}+h^{2} \frac{\bar{b}^{2}-1 / 4}{\operatorname{dist}\left(\Phi(\cdot), \partial \mathbb{R}_{+}^{d}\right)^{2}}+h^{2} V-1\right) \phi\right)_{-}$.

If $\ell$ is sufficiently small so that $C_{0} \omega(4 \ell) \leq 1 / 2$ then $\left(1+C_{0} \omega(4 \ell)\right)^{-1} \geq 1-C_{0} \omega(4 \ell)>0$, and hence

$\operatorname{Tr}\left(\tilde{\phi} H_{\mathbb{R}_{+}^{d}, \bar{b}, \tilde{V}}(h) \tilde{\phi}\right)_{-} \leq \operatorname{Tr}\left(\phi\left(-h^{2}\left(1-C_{0} \omega(4 \ell)\right) \Delta_{\Omega}+h^{2} \frac{\bar{b}^{2}-1 / 4}{\operatorname{dist}\left(\Phi(\cdot), \partial \mathbb{R}_{+}^{d}\right)^{2}}+h^{2} V-1\right) \phi\right)_{-}$.

By splitting into cases depending on the sign of $\bar{b}^{2}-1 / 4$ as in the proof of the upper bound one finds

$$
\begin{aligned}
& \operatorname{Tr}\left(\phi\left(-h^{2}\left(1-C_{0} \omega(4 \ell)\right) \Delta_{\Omega}+h^{2} \frac{\bar{b}^{2}-1 / 4}{\operatorname{dist}\left(\Phi(\cdot), \partial \mathbb{R}_{+}^{d}\right)^{2}}+h^{2} V-1\right) \phi\right)_{-} \\
& \quad \leq \operatorname{Tr}\left(\phi\left(-h^{2}\left(1-C_{0} \omega(4 \ell)\right) \Delta_{\Omega}+h^{2} \frac{\bar{b}^{2}-1 / 4-C \omega(8 \ell)^{2}}{\operatorname{dist}(\cdot, \partial \Omega)^{2}}+h^{2} V-1\right) \phi\right)_{-}
\end{aligned}
$$

for a constant $C$ depending on $d, \bar{b}$.

For any $2 C_{0} \omega(4 \ell)<\rho \leq 1 / 2$ we estimate

$$
\begin{aligned}
\operatorname{Tr}(\phi( & \left.\left.-h^{2}\left(1-C_{0} \omega(4 \ell)\right) \Delta_{\Omega}+h^{2} \frac{\bar{b}^{2}-1 / 4-C \omega(8 \ell)^{2}}{\operatorname{dist}(\cdot, \partial \Omega)^{2}}+h^{2} V-1\right) \phi\right)_{-} \\
\leq & \operatorname{Tr}\left(\phi H_{\Omega, \bar{b}, V}(h) \phi\right)_{-} \\
& \quad+\operatorname{Tr}\left(\phi\left(-h^{2}\left(\rho-C_{0} \omega(4 \ell)\right) \Delta_{\Omega}+h^{2} \frac{\rho\left(\bar{b}^{2}-1 / 4\right)-C \omega(8 \ell)^{2}}{\operatorname{dist}(\cdot, \partial \Omega)^{2}}+h^{2} \rho V-\rho\right)_{-}\right)_{-} \\
\leq & \operatorname{Tr}\left(\phi H_{\Omega, b, V}(h) \phi\right)_{-} \\
& +\operatorname{Tr}\left(\phi\left(-h^{2}\left(\rho-C_{0} \omega(4 \ell) \Delta_{\Omega}+h^{2} \frac{\rho\left(\bar{b}^{2}-1 / 4\right)-C \omega(8 \ell)^{2}}{\operatorname{dist}(\cdot, \partial \Omega)^{2}}+h^{2} \rho V-\rho\right)^{2}\right)_{-} .\right.
\end{aligned}
$$


Provided the analogue of (3.2) with $\bar{b}$ instead of $\underline{b}$ holds we can apply the local HardyLieb-Thirring inequality of Lemma 2.4 to bound

$$
\begin{aligned}
& \operatorname{Tr}\left(\phi\left(-h^{2}\left(\rho-C_{0} \omega(4 \ell)\right) \Delta_{\Omega}+h^{2} \frac{\rho\left(\bar{b}^{2}-1 / 4\right)-C \omega(8 \ell)^{2}}{\operatorname{dist}(\cdot, \partial \Omega)^{2}}+h^{2} \rho V-\rho\right) \phi\right)_{-} \\
& \quad \leq C \rho \ell^{d} h^{-d}\left(1+h^{2}\left\|V_{-}\right\|_{L^{1+d / 2}(\Omega \cap B)}^{1+d / 2}\right) .
\end{aligned}
$$

Again we can set $\rho=\sqrt{\omega(4 \ell)}+\omega(8 \ell)$ and combine the above estimates to arrive at

$$
\begin{aligned}
\operatorname{Tr}\left(\tilde{\phi} H_{\mathbb{R}_{+}^{d}, \bar{b}, \tilde{V}}(h) \tilde{\phi}\right)_{-} \leq & \operatorname{Tr}\left(\phi H_{\Omega, b, V}(h) \phi\right)_{-} \\
& +C \ell^{d} h^{-d}(\sqrt{\omega(4 \ell)}+\omega(8 \ell))\left(1+h^{2}\left\|V_{-}\right\|_{L^{1+d / 2}(\Omega \cap B)}^{1+d / 2}\right) .
\end{aligned}
$$

This completes the proof of the lower bound and hence the proof of Lemma 3.3.

The proof of Theorem 3.2 has been reduced to understanding the asymptotics of $\operatorname{Tr}\left(\phi H_{\mathbb{R}_{+}^{d}, b, V}(h) \phi\right)_{-}$with $b(x) \equiv b>0$.

Lemma 3.4. Let $\Omega, V$ be as in Theorem 1.1. Let $\phi \in C_{0}^{1}\left(\mathbb{R}^{d}\right)$ be supported in a ball $\bar{B}$ of radius $\ell$ and satisfy

$$
\|\nabla \phi\|_{L^{\infty}} \leq M \ell^{-1} .
$$

With $b(x) \equiv b>0$ we have, for $0<h \leq K \ell$,

$$
\begin{gathered}
\left|\operatorname{Tr}\left(\phi H_{\mathbb{R}_{+}^{d}, b, V}(h) \phi\right)_{-}-L_{d} h^{-d} \int_{\mathbb{R}_{+}^{d}} \phi^{2}(y) d y+\frac{b L_{d-1}}{2} h^{-d+1} \int_{\partial \mathbb{R}_{+}^{d}} \phi^{2}(y) d \mathcal{H}^{d-1}(y)\right| \\
\leq C h^{-d+2}\left(\ell^{d-2}|\log (\ell / h)|+\left\|V_{+}\right\|_{L^{1}\left(\mathbb{R}_{+}^{d} \cap B\right)}+\ell^{d}\left\|V_{-}\right\|_{L^{1+d / 2}\left(\mathbb{R}_{+}^{d} \cap B\right)}^{1+d / 2}\right),
\end{gathered}
$$

where $C$ depends only on $d, M, K, b$ and can be uniformly bounded for $b$ in compact subsets of $[0, \infty)$.

Proof. Our proof proceeds by diagonalizing the operator $H_{\mathbb{R}_{+}^{d}, b, 0}(h)$. For the general background on what follows, see [4, Chapter XIII].

For $f \in C^{2}\left(\mathbb{R}_{+}\right)$define the differential expression

$$
L_{b} f(x)=f^{\prime \prime}(x)-\left(b^{2}-\frac{1}{4}\right) \frac{f(x)}{x^{2}} .
$$

The operator $H_{\mathbb{R}_{+}^{d}, b, 0}(h)$ can then be decomposed as

$$
H_{\mathbb{R}_{+}^{d}, b, 0}(h)=-h^{2} \Delta^{\prime}-h^{2} L_{b},
$$

where $\Delta^{\prime}=\sum_{j=1}^{d-1} \frac{\partial^{2}}{\partial y_{j}^{2}}$ and $L_{b}$ acts in the $y_{d}$-coordinate. 
For $b>0, \mu \geq 0$ the ODE

$$
-L_{b} u(x)=\mu u(x)
$$

has two linearly independent solutions

$$
\psi_{b, \mu}(x)=x^{1 / 2} J_{b}(x \sqrt{\mu}) \quad \text { and } \quad \eta_{b, \mu}(x)=x^{1 / 2} Y_{b}(x \sqrt{\mu}) .
$$

If $b \geq 1 / 2$ only $\psi$ vanishes at $x=0$ while for $b \in(0,1 / 2)$ both solutions vanish, indeed $\psi \sim x^{1 / 2+b}$ and $\eta \sim x^{1 / 2-b}$ as $x \rightarrow 0^{+}$. However, for any $b \neq \frac{1}{2}$ only the first solution $\psi_{b, v}$ is in $H^{1}$ around zero. In particular, our effective operator $H_{\mathbb{R}_{+}^{d}, b, 0}(h)$ is diagonalized through a Fourier transform with respect to $y^{\prime}$ and a Hankel transform $\mathfrak{S}_{b}$ with respect to $y_{d}$. Recall that the Hankel transform $\mathfrak{S}_{\alpha}: L^{2}\left(\mathbb{R}_{+}\right) \rightarrow L^{2}\left(\mathbb{R}_{+}\right)$is initially defined by

$$
\mathfrak{H}_{\alpha}(g)(s)=\int_{0}^{\infty} g(t) J_{\alpha}(s t) \sqrt{s t} d t \quad \text { for } g \in L^{1}\left(\mathbb{R}_{+}\right)
$$

and extended to $L^{2}\left(\mathbb{R}_{+}\right)$in a similar manner as the Fourier transform. Moreover, $\mathfrak{H}_{\alpha}$ is unitary, is its own inverse $\mathfrak{S}_{\alpha}^{2}=1$. Moreover, for $G \in L^{\infty}\left(\mathbb{R}_{+}\right)$with compact support and $f \in H_{0}^{1}\left(\mathbb{R}_{+}\right) \cap H^{2}\left(\mathbb{R}_{+}\right)$

$$
\left\langle f, G\left(-L_{b}\right) f\right\rangle_{L^{2}\left(\mathbb{R}_{+}\right)}=\int_{0}^{\infty} G\left(s^{2}\right)\left|\mathfrak{H}_{b}(f)(s)\right|^{2} d s .
$$

By a similar argument as in the proof of Lemma 2.4 the upper bound can be reduced to the case $V \equiv 0$. Indeed, for any $0<\rho \leq 1 / 2$,

$$
\begin{aligned}
\operatorname{Tr}(\phi & \left.H_{\mathbb{R}_{+}^{d}, b, V}(h) \phi\right)_{-} \\
& \leq \operatorname{Tr}\left(\phi H_{\mathbb{R}_{+}^{d}, b, 0}(h(1-\rho)) \phi\right)_{-}+\operatorname{Tr}\left(\phi\left(h^{2} \rho \Delta_{\mathbb{R}_{+}^{d}}+h^{2} \rho \frac{b^{2}-1 / 4}{\operatorname{dist}\left(\cdot, \partial \mathbb{R}_{+}^{d}\right)^{2}}-h^{2} V\right) \phi\right)_{-} \\
& \leq \operatorname{Tr}\left(\phi H_{\mathbb{R}_{+}^{d}, b, 0}(h(1-\rho)) \phi\right)_{-}+C h^{2} \rho^{-d / 2}\left\|V_{-}\right\|_{L^{1+d / 2}\left(\mathbb{R}_{+}^{d} \cap B\right)}^{1+d / 2} .
\end{aligned}
$$

Set $\rho=h^{2} /\left(2 K^{2} \ell^{2}\right)$ so that $h^{2} \rho^{-d / 2}=O\left(\ell^{d} h^{-d+2}\right)$ and $(h(1-\rho))^{-\beta}=h^{-\beta}(1+$ $\left.O\left(\ell^{-2} h^{2}\right)\right)$. The claimed upper bound now follows from the case $V \equiv 0$.

Using the inequality $\operatorname{Tr}(\phi H \phi)_{-} \leq \operatorname{Tr}\left(\phi H_{-} \phi\right)$, applying the Fourier transform with respect to $y^{\prime}$ and the Hankel transform in the $y_{d}$-direction yields

$$
\begin{aligned}
\operatorname{Tr}\left(\phi H_{\mathbb{R}_{+}^{d}, b, 0}(h) \phi\right)_{-} & \leq \operatorname{Tr}\left(\phi\left(H_{\mathbb{R}_{+}^{d}, b, 0}(h)\right)_{-} \phi\right) \\
& =\frac{1}{(2 \pi)^{d-1}} \iint_{\mathbb{R}_{+}^{d} \times \mathbb{R}_{+}^{d}} \phi^{2}(y)\left(h^{2}|\xi|^{2}-1\right)_{-} \xi_{d} y_{d} J_{b}\left(\xi_{d} y_{d}\right)^{2} d \xi d y .
\end{aligned}
$$

For the lower bound define the operator $\gamma$ with integral kernel

$$
\gamma(x, y)=\frac{1}{(2 \pi)^{d-1}} \chi(x) \int_{\mathbb{R}_{+}^{d} \cap B_{h^{-1}}(0)} e^{i \xi^{\prime}\left(x^{\prime}-y^{\prime}\right)} \sqrt{\xi_{d} x_{d}} J_{b}\left(\xi_{d} x_{d}\right) \sqrt{\xi_{d} y_{d}} J_{b}\left(\xi_{d} y_{d}\right) d \xi \chi(y),
$$


where $\chi \in C_{0}^{\infty}\left(\mathbb{R}^{d}\right)$ is such that $0 \leq \chi \leq 1$ and $\chi \equiv 1$ on supp $\phi$. The operator $\gamma$ is trace class, satisfies $0 \leq \gamma \leq \mathbf{1}$, and its range is contained in the domain of $H_{\mathbb{R}_{+}^{d}, b, V}$. Thus, by the variational principle,

$$
\begin{aligned}
&-\operatorname{Tr}\left(\phi H_{\mathbb{R}_{+}^{d}, b, V}(h) \phi\right)_{-} \\
& \leq \operatorname{Tr}\left(\gamma \phi H_{\mathbb{R}_{+}^{d}, b, V_{+}}(h) \phi\right) \\
&= \frac{1}{(2 \pi)^{d-1}} \iint_{\mathbb{R}_{+}^{d} \times \mathbb{R}_{+}^{d}}\left(h^{2}|\xi|^{2}-1\right)_{-} \phi^{2}(x) \xi_{d} x_{d} J_{b}\left(\xi_{d} x_{d}\right)^{2} d \xi d x \\
&+h^{-d+2} \int_{\mathbb{R}_{+}^{d}}\left(V_{+}(x) \phi^{2}(x)+|\nabla \phi(x)|^{2}\right) \int_{0}^{1}\left(x_{d} t h^{-1}\right) J_{b}\left(x_{d} t h^{-1}\right)^{2} d t d x \\
& \leq \frac{1}{(2 \pi)^{d-1}} \iint_{\mathbb{R}_{+}^{d} \times \mathbb{R}_{+}^{d}}\left(h^{2}|\xi|^{2}-1\right)_{-} \phi^{2}(x) \xi_{d} x_{d} J_{b}\left(\xi_{d} x_{d}\right)^{2} d \xi d x \\
&+C h^{-d+2} \int_{\mathbb{R}_{+}^{d}}\left(V_{+}(x) \phi^{2}(x)+|\nabla \phi(x)|^{2}\right) d x,
\end{aligned}
$$

with $C$ uniformly bounded for $b$ in compact subsets of $[0, \infty)$, since $\left\|\sqrt{\cdot} J_{b}\right\|_{L^{\infty}\left(\mathbb{R}_{+}\right)}<\infty$ uniformly for $b$ in compact subsets of $[0, \infty)$ (see [12, Chapter 7]). By (3.3) we can estimate $\|\phi\|_{L^{\infty}} \leq M$ and $\int_{\mathbb{R}_{+}^{d}}|\nabla \phi(x)|^{2} d x \leq C \ell^{d-2}$.

What remains is to understand the common integral in (3.4) and (3.5). We begin by extracting the desired leading term:

$$
\begin{aligned}
& \frac{1}{(2 \pi)^{d-1}} \iint_{\mathbb{R}_{+}^{d} \times \mathbb{R}_{+}^{d}} \phi^{2}(y)\left(h^{2}|\xi|^{2}-1\right)_{-} \xi_{d} y_{d} J_{b}\left(\xi_{d} y_{d}\right)^{2} d \xi d y \\
& =L_{d} h^{-d} \int_{\mathbb{R}_{+}^{d}} \phi^{2}(y) d y \\
& \quad-L_{d-1} h^{-d+1} \int_{0}^{\infty} \int_{\mathbb{R}^{d-1}} \phi^{2}\left(y^{\prime}, h t\right) d y^{\prime} \int_{0}^{1}\left(1-\xi_{d}^{2}\right)^{(d+1) / 2}\left(\frac{1}{\pi}-\xi_{d} t J_{b}\left(\xi_{d} t\right)^{2}\right) d \xi_{d} d t .
\end{aligned}
$$

Define, for $b \geq 0$ and $t \geq 0$,

$$
P_{b}(t)=\int_{0}^{1}\left(1-\xi^{2}\right)^{(d+1) / 2}\left(\frac{1}{\pi}-\xi t J_{b}(\xi t)^{2}\right) d \xi
$$

In Lemmas A.1 and A.2 we shall prove that

$$
\int_{0}^{\infty} P_{b}(t) d t=\frac{b}{2} \quad \text { and } \quad P_{b}(t)=O\left(t^{-2}\right) \text { as } t \rightarrow \infty,
$$

with the implicit constant uniformly bounded for $b$ in compact subsets of $[0, \infty)$. 
Using (3.7) we can estimate

$$
\begin{aligned}
& \int_{0}^{\infty} \int_{\mathbb{R}^{d-1}} \phi^{2}\left(y^{\prime}, h t\right) d y^{\prime} P_{b}(t) d t \\
&=\int_{0}^{2 \ell / h} \int_{\mathbb{R}^{d-1}} \phi^{2}\left(y^{\prime}, h t\right) d y^{\prime} P_{b}(t) d t \\
&=\frac{b}{2} \int_{\mathbb{R}^{d-1}} \phi^{2}\left(y^{\prime}, 0\right) d y^{\prime}-\int_{2 \ell / h}^{\infty} \int_{\mathbb{R}^{d-1}} \phi^{2}\left(y^{\prime}, 0\right) d y^{\prime} P_{b}(t) d t \\
&+2 \int_{0}^{2 \ell / h} h t \int_{\mathbb{R}^{d-1}} \int_{0}^{1} \phi\left(y^{\prime}, h t s\right) \partial_{y_{d}} \phi\left(y^{\prime}, h t s\right) d s d y^{\prime} P_{b}(t) d t \\
&= \frac{b}{2} \int_{\mathbb{R}^{d-1}} \phi^{2}\left(y^{\prime}, 0\right) d y^{\prime}+O\left(h \ell^{d-2}|\log (\ell / h)|\right) .
\end{aligned}
$$

Combined with (3.6), (3.4), and (3.5) this completes the proof of Lemma 3.4.

We are now ready to prove Theorem 3.2.

Proof of Theorem 3.2. By combining Lemma 3.3 and Lemma 3.4 the claimed estimate follows from

$$
\begin{aligned}
\int_{\partial \Omega} \phi^{2}(x) & {\left[b(x)-\inf _{y \in \Omega \cap B} b(y)\right] d \mathcal{H}^{d-1}(x) } \\
& \leq \int_{\partial \Omega} \phi^{2}(x)\left[\sup _{y \in \Omega \cap B} b(y)-\inf _{y \in \Omega \cap B} b(y)\right] d \mathcal{H}^{d-1}(x),
\end{aligned}
$$

and the corresponding inequality for the sup and the fact that $\operatorname{supp} \phi \subseteq \bar{B} \subset \overline{B_{2 \ell}(x)}$ for any $x \in \operatorname{supp} \phi$.

\section{From local to global asymptotics}

In this section we prove our main result by piecing together the local asymptotics obtained above. The key ingredient is the following construction of a continuum partition of unity due to Solovej and Spitzer [11].

Let

$$
\ell(u)=\frac{1}{2} \max \left\{\operatorname{dist}\left(u, \Omega^{c}\right), 2 \ell_{0}\right\}
$$

with a small parameter $0<\ell_{0}$ to be determined. Note that $0<\ell \leq \max \left\{\frac{r_{\text {in }}(\Omega)}{2}, \ell_{0}\right\}$ and, since $\left|\nabla \operatorname{dist}\left(u, \Omega^{c}\right)\right|=1$ a.e., $\|\nabla \ell\|_{L^{\infty}} \leq \frac{1}{2}$. Note also that $\left.\operatorname{dist}\left(B_{\ell(u)}, \Omega^{c}\right)\right) \leq 2 \ell(u)$ if and only if $\operatorname{dist}(u, \partial \Omega) \leq 2 \ell_{0}$ in which case $\ell(u)=\ell_{0}$. In particular, if $\operatorname{dist}(u, \Omega)>\ell_{0}$ then $B_{\ell(u)}(u) \cap \Omega=\emptyset$. 
Fix a function $\phi \in C_{0}^{\infty}\left(\mathbb{R}^{d}\right)$ with $\operatorname{supp} \phi \subseteq \overline{B_{1}(0)}$ and $\|\phi\|_{L^{2}}=1$. By [11, Theorem 22] (see also [7, Lemma 2.5]) the functions

$$
\phi_{u}(x)=\phi\left(\frac{x-u}{\ell(u)}\right) \sqrt{1+\nabla \ell(u) \cdot \frac{x-y}{\ell(u)}}, \quad x \in \mathbb{R}^{d}, u \in \mathbb{R}^{d},
$$

belong to $C_{0}^{\infty}\left(\mathbb{R}^{d}\right)$ with supp $\phi_{u} \subseteq \overline{B_{\ell(u)}(u)}$, satisfy

$$
\int_{\mathbb{R}^{d}} \phi_{u}(x)^{2} \ell(u)^{-d} d u=1 \quad \text { for all } x \in \mathbb{R}^{d}
$$

and, with a constant $C$ depending only on $d$,

$$
\left\|\phi_{u}\right\|_{L^{\infty}} \leq \sqrt{2}\|\phi\|_{L^{\infty}} \quad \text { and } \quad\left\|\nabla \phi_{u}\right\|_{L^{\infty}} \leq C \ell(u)^{-1}\|\nabla \phi\|_{L^{\infty}} \quad \text { for all } u \in \mathbb{R}^{d} .
$$

The application to our problem here is summarized in the following lemma:

Lemma 4.1. Let $\Omega, b, V$ be as in Theorem 1.1 and define $\ell,\left\{\phi_{u}\right\}_{u \in \mathbb{R}^{d}}$ as above. Then, for $0<\ell_{0} \leq c(\Omega, b)$ and $0<h \leq K \ell_{0}$,

$$
\begin{aligned}
\mid \operatorname{Tr}\left(H_{\Omega, b, V}(h)\right)_{-} & -\int_{\mathbb{R}^{d}} \operatorname{Tr}\left(\phi_{u} H_{\Omega, b, V}(h) \phi_{u}\right)_{-} \ell(u)^{-d} d u \mid \\
& \leq C h^{-d+2} \int_{\operatorname{dist}(u, \Omega) \leq \ell_{0}}\left(1+h^{2}\left\|V_{-}\right\|_{L^{1+d / 2}\left(\Omega \cap B_{\ell(u)}(u)\right)}^{1+d / 2}\right) \ell(u)^{-2} d u,
\end{aligned}
$$

where the constant $C$ depends only on $\Omega, b, K,\|\phi\|_{L^{\infty}}$.

For the sake of brevity, we omit the proof of Lemma 4.1 and instead refer the reader to the proof of [7, Lemma 2.8]. Lemma 4.1 can be proved in the same manner but replacing the use of a local Berezin-Li-Yau inequality by an application of Lemma 2.4.

With the above results in hand we are ready to prove Theorem 1.1.

Proof of Theorem 1.1. Set $\ell_{0}=h / \varepsilon_{0}$ with $0<h \leq \varepsilon_{0} r_{i n}(\Omega) / 2$ for a parameter $\varepsilon_{0} \in$ $(0,1]$ which will eventually tend to zero.

We divide the set of $u \in \mathbb{R}^{d}$ such that $B_{\ell(u)}(u) \cap \Omega \neq \emptyset$ into two disjoint parts:

$$
\Omega_{*}=\left\{u \in \mathbb{R}^{d}: 2 \ell_{0}<\delta_{\Omega}(u)\right\} \quad \text { and } \quad \Omega^{*}=\left\{u \in \mathbb{R}^{d}:-\ell_{0}<\delta_{\Omega}(u) \leq 2 \ell_{0}\right\},
$$

where $\delta_{\Omega}$ denotes the signed distance function to the boundary, $\delta_{\Omega}(y)=\operatorname{dist}\left(u, \Omega^{c}\right)-$ $\operatorname{dist}(u, \Omega)$. Note that for all $u \in \Omega^{*}$ we have $\ell(u)=\ell_{0}$.

By Lemma 4.1 we need to understand the integral with respect to $u$ of the local traces $\operatorname{Tr}\left(\phi_{u} H_{\Omega, b, V}(h) \phi_{u}\right)_{-}$. Breaking the integral according to the partition (4.2) we have

$$
\begin{aligned}
\int_{\mathbb{R}^{d}} \operatorname{Tr}\left(\phi_{u} H_{\Omega, b, V}(h) \phi_{u}\right)_{-} \ell(u)^{-d} d u= & \int_{\Omega_{*}} \operatorname{Tr}\left(\phi_{u} H_{\Omega, b, V}(h) \phi_{u}\right)_{-} \ell(u)^{-d} d u \\
& +\int_{\Omega^{*}} \operatorname{Tr}\left(\phi_{u} H_{\Omega, b, V}(h) \phi_{u}\right)_{-} \ell_{0}^{-d} d u .
\end{aligned}
$$


For the first term Lemma 3.1 with $V_{0}(x)=\frac{\left(b(x)^{2}-1 / 4\right)_{-}}{\operatorname{dist}(x, \partial \Omega)^{2}}, V_{1}=V_{-}(x)$ yields

$$
\begin{aligned}
& \int_{\Omega_{*}} \operatorname{Tr}\left(\phi_{u} H_{\Omega, b, V}(h) \phi_{u}\right)_{-} \ell(u)^{-d} d u \\
& \quad=L_{d} h^{-d} \int_{\Omega_{*}} \int_{\Omega} \phi_{u}^{2}(x) \ell(u)^{-d} d x d u \\
& \quad+O\left(h^{-d+2}\right) \int_{\Omega_{*}}\left[\ell(u)^{-2}\left(1+\|b\|_{L^{\infty}}^{2}\right)+\left\|V_{-}\right\|_{L^{1+d / 2}\left(B_{\ell(u)}(u)\right)}^{1+d / 2}+\ell(u)^{-d}\left\|V_{+}\right\|_{L^{1}\left(B_{\ell(u)}(u)\right)}\right] d u
\end{aligned}
$$

where we used $\left\|V_{0}\right\|_{L^{\infty}} \leq \frac{C}{(\operatorname{dist}(u, \partial \Omega)-\ell(u))^{2}} \leq C \ell(u)^{-2}$ and $\frac{\left(b(x)^{2}-1 / 4\right)_{+}}{\operatorname{dist}(x, \partial \Omega)^{2}} \leq C\|b\|_{L^{\infty}}^{2} \ell(u)^{-2}$.

For the integral over the boundary region $\Omega^{*}$ Theorem 3.2, for $\varepsilon_{0}, \ell_{0}, h$ sufficiently small, implies

$$
\begin{aligned}
\int_{\Omega^{*}} & \operatorname{Tr}\left(\phi_{u} H_{\Omega, b, V}(h) \phi_{u}\right)_{-} \ell_{0}^{-d} d u \\
= & L_{d} h^{-d} \int_{\Omega^{*}} \int_{\Omega} \phi_{u}^{2}(x) \ell_{0}^{-d} d x d u-\frac{L_{d-1}}{2} h^{-d+1} \int_{\Omega^{*}} \int_{\partial \Omega} \phi_{u}^{2}(x) b(x) \ell_{0}^{-d} d \mathcal{H}^{d-1}(x) d u \\
& +O\left(h^{-d}\right)\left|\Omega^{*}\right|\left(o_{\ell_{0} \rightarrow 0^{+}}(1)+\varepsilon_{0}^{2}\left|\log \left(\varepsilon_{0}\right)\right|\right)+h^{-d+1} o_{\ell_{0} \rightarrow 0^{+}}(1) \\
& +O\left(h^{-d+2}\right) \int_{\Omega^{*}}\left[\left\|V_{-}\right\|_{L^{1+d / 2}\left(B_{\ell(u)}(u)\right)}^{1+d / 2}+\ell_{0}^{-d}\left\|V_{+}\right\|_{L^{1}\left(B_{\ell(u)}(u)\right)}\right] d u .
\end{aligned}
$$

Here we used the fact that $b$ satisfies (1.3).

Combining the estimates for the contribution from the bulk and boundary region, using (4.1), and estimating the integrals of the norms of $V_{-}, V_{+}$, we find

$$
\begin{aligned}
\int_{\mathbb{R}^{d}} & \operatorname{Tr}\left(\phi_{u} H_{\Omega, b, V}(h) \phi_{u}\right)_{-} \ell(u)^{-d} d u \\
= & L_{d} h^{-d}|\Omega|-\frac{L_{d-1}}{2} h^{-d+1} \int_{\partial \Omega} b(x) d \mathcal{H}^{d-1}(x) \\
& +O\left(h^{-d}\right)\left|\Omega^{*}\right|\left(o \ell_{0} \rightarrow 0^{+}(1)+\varepsilon_{0}^{2}\left|\log \left(\varepsilon_{0}\right)\right|\right)+h^{-d+1} o \ell_{0} \rightarrow 0^{+}(1) \\
& +O\left(h^{-d+2}\right)\left(1+\|b\|_{L^{\infty}}^{2}\right) \int_{\Omega_{*}} \ell(u)^{-2} d u+O\left(h^{-d+2}\right)\left[\left\|V_{-}\right\|_{L^{1+d / 2}(\Omega)}^{1+d / 2}+\left\|V_{+}\right\|_{L^{1}(\Omega)}\right] .
\end{aligned}
$$

By [7, eq.'s (4.6)-(4.8)], $\int_{\Omega_{*}} \ell(u)^{-2} d u \leq C \ell_{0}^{-1}$ and $\left|\Omega^{*}\right| \leq C \ell_{0}$ with $C$ depending only on $\Omega$. Thus by Lemma 4.1, (4.3), and since $h^{2} / \ell(u)^{2} \leq \varepsilon_{0}^{2}$ we conclude that

$$
\begin{aligned}
& h^{d-1}\left|\operatorname{Tr}\left(H_{\Omega, b, V}(h)\right)_{-}-L_{d} h^{-d}\right| \Omega\left|+\frac{L_{d-1}}{2} h^{-d+1} \int_{\partial \Omega} b(x) d \mathcal{H}^{d-1}(x)\right| \\
& \leq \varepsilon_{0}^{-1} o_{h / \varepsilon_{0} \rightarrow 0^{+}}(1)+O\left(\varepsilon_{0}\left|\log \left(\varepsilon_{0}\right)\right|\right)+o_{h / \varepsilon_{0} \rightarrow 0^{+}}(1) \\
&+O\left(\varepsilon_{0}\right)\left(1+\|b\|_{L^{\infty}}^{2}\right)+O(h)\left[\left\|V_{-}\right\|_{L^{1+d / 2}(\Omega)}^{1+d / 2}+\left\|V_{+}\right\|_{L^{1}(\Omega)}\right] .
\end{aligned}
$$

Letting first $h$ and then $\varepsilon_{0}$ tend to 0 completes the proof of Theorem 1.1. 


\section{A Properties of $P_{v}$}

Our aim is to prove the following two lemmas.

Lemma A.1. For $v \geq 0$ it holds that

$$
P_{v}(t)=\int_{0}^{1}\left(1-\xi^{2}\right)^{(d+1) / 2}\left(\frac{1}{\pi}-\xi t J_{v}(\xi t)^{2}\right) d \xi=O\left(t^{-2}\right) \quad \text { as } t \rightarrow \infty .
$$

Moreover, the implicit constant is uniformly bounded for $v$ in compact subsets of $[0, \infty)$.

Lemma A.2. For any $v \geq 0$ we have the identity

$$
\int_{0}^{\infty} P_{v}(t) d t=\int_{0}^{\infty} \int_{0}^{1}\left(1-\xi^{2}\right)^{(d+1) / 2}\left(\frac{1}{\pi}-\xi t J_{v}(\xi t)^{2}\right) d \xi d t=\frac{v}{2} .
$$

We shall need the following asymptotic expansion for the Bessel function

$$
J_{v}(t)=\left(\frac{2}{\pi t}\right)^{1 / 2}\left[\cos \left(t-\frac{v \pi}{2}-\frac{\pi}{4}\right)-\frac{4 v^{2}-1}{8 t} \sin \left(t-\frac{v \pi}{2}-\frac{\pi}{4}\right)+O\left(t^{-2}\right)\right],
$$

where the implicit constant is uniformly bounded for $v$ in compact subsets of $[0, \infty)$ (see [12, Chapter 7]). We shall also make use of the following identity

$$
x J_{v}(x)^{2}=\frac{d}{d x}\left[\frac{x^{2}}{2} J_{v}(x)^{2}+\frac{x^{2}}{2} J_{v+1}(x)^{2}-v x J_{v}(x) J_{v+1}(x)\right],
$$

which is easily deduced from $J_{v}^{\prime}(x)=\frac{1}{2}\left(J_{v-1}(x)-J_{v+1}(x)\right)$ and the recursion formula $J_{v-1}(x)+J_{v+1}(x)=\frac{2 v}{x} J_{v}(x)$.

Proof of Lemma A.1. By an integration by parts, (A.2), and since $\left|J_{v}(x)\right| \leq 1$,

$$
\begin{aligned}
P_{v}(t)=(d+1) \int_{\delta}^{1}\left(1-\xi^{2}\right)^{(d-1) / 2}\left[\frac{\xi^{2}}{\pi}\right. & -\frac{t \xi^{3}}{2} J_{v}(\xi t)^{2}-\frac{t \xi^{3}}{2} J_{v+1}(\xi t)^{2} \\
& \left.+v \xi^{2} J_{v}(\xi t) J_{v+1}(\xi t)\right] d \xi+O\left(t \delta^{4}+\delta^{3}\right)
\end{aligned}
$$

for any $0 \leq \delta<1$. Provided $\delta t \gtrsim 1$, (A.1) implies

$$
\frac{\xi^{2}}{\pi}-\frac{t \xi^{3}}{2} J_{v}(\xi t)^{2}-\frac{t \xi^{3}}{2} J_{v+1}(\xi t)^{2}+v \xi^{2} J_{v}(\xi t) J_{v+1}(\xi t)=\frac{\xi}{2 \pi t} \cos (2 \xi t-\pi v)+O\left(t^{-2}\right),
$$

with the implicit constant uniformly bounded for $v$ in compact subsets of $[0, \infty)$. Thus, we have arrived at

$$
\begin{aligned}
P_{v}(t) & =\frac{d+1}{2 \pi t} \int_{\delta}^{1}\left(1-\xi^{2}\right)^{(d-1) / 2} \xi \cos (2 \xi t-\pi v) d \xi+O\left(t^{-2}+t \delta^{4}+\delta^{3}\right) \\
& =\frac{d+1}{2 \pi t} \int_{0}^{1}\left(1-\xi^{2}\right)^{(d-1) / 2} \xi \cos (2 \xi t-\pi v) d \xi+O\left(t^{-2}\right)
\end{aligned}
$$


where we chose $\delta=O\left(t^{-1}\right)$. An integration by parts yields

$\int_{0}^{1}\left(1-\xi^{2}\right)^{(d-1) / 2} \xi \cos (2 \xi t-\pi v) d \xi=\frac{1}{2 t} \int_{0}^{1}\left(1-\xi^{2}\right)^{(d-3) / 2}\left(d \xi^{2}-1\right) \sin (2 \xi t-\pi v) d \xi$.

Since the integral on the right is bounded uniformly in $v$, this completes the proof.

Proof of Lemma A.2. For any $T>0$, by (A.2), Fubini's theorem, and a change of variables

$$
\begin{aligned}
\int_{0}^{T} P_{v}(t) d t & =\int_{0}^{1}\left(1-\xi^{2}\right)^{(d+1) / 2} \int_{0}^{T}\left(\frac{1}{\pi}-\xi t J_{v}(\xi t)^{2}\right) d t d \xi \\
& =\frac{T}{2}\left(P_{v}(T)+P_{v+1}(T)\right)+v \int_{0}^{T}\left(1-s^{2} / T^{2}\right)^{(d+1) / 2} J_{v}(s) J_{v+1}(s) d s .
\end{aligned}
$$

By Lemma A.1 only the remaining integral contributes as $T \rightarrow \infty$. By [12, p. 406] and for $v>-1$, in the sense of an improper Riemann integral

$$
\int_{0}^{\infty} J_{v}(s) J_{v+1}(s) d s=\frac{1}{2} .
$$

The proof is completed by appealing to a simple Abelian theorem in Lemma A.3.

Lemma A.3. If $f \in L^{\infty}\left(\mathbb{R}_{+}\right)$and $\lim _{T \rightarrow \infty} \int_{0}^{T} f(t) d t=A$, then for all $\alpha>0$

$$
\lim _{T \rightarrow \infty} \int_{0}^{T}\left(1-\frac{t^{2}}{T^{2}}\right)^{\alpha} f(t) d t=A .
$$

Proof. By integration by parts and a change of variables,

$$
\begin{aligned}
\int_{0}^{T}\left(1-\frac{t^{2}}{T^{2}}\right)^{\alpha} f(t) d t & =\int_{0}^{T}\left(-\frac{d}{d t}\left(1-\frac{t^{2}}{T^{2}}\right)^{\alpha}\right) \int_{0}^{t} f(s) d s d t \\
& =2 \alpha \int_{0}^{1}\left(1-\sigma^{2}\right)^{\alpha-1} \sigma \int_{0}^{\sigma T} f(s) d s d \sigma
\end{aligned}
$$

By our assumptions there is a $S_{0}<\infty$ so that for $S \geq S_{0}$

$$
\left|\int_{0}^{S} f(s) d s\right| \leq|A|+1
$$

Since $f$ is bounded,

$$
\left|\int_{0}^{S} f(s) d s\right| \leq S\|f\|_{\infty}
$$

Thus, for all $\sigma, T$,

$$
\left|\int_{0}^{\sigma T} f(s) d s\right| \leq \max \left\{|A|+1, S_{0}\|f\|_{\infty}\right\} .
$$


Since $\alpha>0$, the function $\sigma \mapsto\left(1-\sigma^{2}\right)^{\alpha-1} \sigma$ is integrable and by dominated convergence,

$$
\lim _{T \rightarrow \infty} 2 \alpha \int_{0}^{1}\left(1-\sigma^{2}\right)^{\alpha-1} \sigma \int_{0}^{\sigma T} f(s) d s d \sigma=2 \alpha A \int_{0}^{1}\left(1-\sigma^{2}\right)^{\alpha-1} \sigma d \sigma=A .
$$

This completes the proof of Lemma A.3.

Acknowledgments. U.S. National Science Foundation grants DMS-1363432 and DMS-1954995 (R.L.F.) and Knut and Alice Wallenberg Foundation grant KAW 2018.0281 (S.L.) are acknowledged.

\section{References}

[1] M. Š. Birman and M. Z. Solomjak, Asymptotic properties of the spectrum of differential equations, J. Soviet Math. 12 (1979), no. 3, 247-283.

[2] M. Š. Birman and M. Z. Solomjak, Quantitative analysis in Sobolev imbedding theorems and applications to spectral theory, Amer. Math. Soc., Providence, R.I., 1980.

[3] B. Davies, The Hardy constant, Quart. J. Math. Oxford 46 (1995), no. 2, 417-431.

[4] N. Dunford and J. T. Schwartz, Linear operators. Part II, Wiley, New York, 1963.

[5] R. L. Frank and L. Geisinger, Two-term spectral asymptotics for the Dirichlet Laplacian on a bounded domain, Mathematical results in quantum physics, World Sci. Publ., Hackensack, NJ, 2011, pp. 138-147.

[6] R. L. Frank and L. Geisinger, Semi-classical analysis of the Laplace operator with Robin boundary conditions, Bull. Math. Sci. 2 (2012), no. 2, 281-319.

[7] R. L. Frank and S. Larson, Two-term spectral asymptotics for the Dirichlet Laplacian in a Lipschitz domain, J. Reine Angew. Math. 766 (2020), 195-228.

[8] R. L. Frank and S. Larson, On the error in the two-term Weyl formula for the Dirichlet Laplacian, J. Math. Phys. 61 (2020), 043504.

[9] R. L. Frank and M. Loss, Hardy-Sobolev-Maz'ya inequalities for arbitrary domains, J. Math. Pures Appl. (9) 97 (2012), no. 1, 39-54.

[10] E. H. Lieb and B. Simon, The Thomas-Fermi theory of atoms, molecules and solids, Adv. Math. 23 (1977), no. 1, 22-116.

[11] J. P. Solovej and W. L. Spitzer, A new coherent states approach to semiclassics which gives Scott's correction, Comm. Math. Phys. 241 (2003), no. 2-3, 383-420.

[12] G. N. Watson, A Treatise on the Theory of Bessel Functions, Cambridge University Press, Cambridge, England, 1944. 